\title{
Breast-Cancer Adjuvant Therapy with Zoledronic Acid
}

\author{
Robert E. Coleman, M.B., B.S., M.D., Helen Marshall, M.Sc., \\ David Cameron, M.B., B.S., M.D., David Dodwell, M.B., Ch.B., M.D., \\ Roger Burkinshaw, B.Sc., Maccon Keane, M.D., Miguel Gil, M.D., \\ Stephen J. Houston, M.B., B.S., M.D., Robert J. Grieve, M.B., Ch.B., \\ Peter J. Barrett-Lee, M.B., B.S., M.D., Diana Ritchie, M.B., Ch.B., M.D., \\ Julia Pugh, C.I.M.Dip., Claire Gaunt, B.Sc., Una Rea, B.Sc., \\ Jennifer Peterson, B.App.Sc., Claire Davies, B.Sc., Victoria Hiley, B.Sc., \\ Walter Gregory, Ph.D., and Richard Bell, M.B., B.S., for the AZURE Investigators*
}

ABSTRACT

The authors' affiliations are listed in the Appendix. Address reprint requests to Dr. Coleman at the Academic Unit of Clinical Oncology, Weston Park Hospital, Sheffield S10 2SJ, United Kingdom, or at r.e.coleman@sheffield.ac.uk.

*Additional investigators in the Adjuvant Zoledronic Acid to Reduce Recurrence (AZURE) trial are listed in the Supplementary Appendix, available at NEJM .org.

This article (10.1056/NEJMoal105195) was published on September 25, 2011, at NEJM.org.

N Engl J Med 2011;365:1396-1405.

Copyright $\odot 2011$ Massachusetts Medical Society.

\section{BACKGROUND}

Data suggest that the adjuvant use of bisphosphonates reduces rates of recurrence and death in patients with early-stage breast cancer. We conducted a study to determine whether treatment with zoledronic acid, in addition to standard adjuvant therapy, would improve disease outcomes in such patients.

\section{METHODS}

In this open-label phase 3 study, we randomly assigned 3360 patients to receive standard adjuvant systemic therapy either with or without zoledronic acid. The zoledronic acid was administered every 3 to 4 weeks for 6 doses and then every 3 to 6 months to complete 5 years of treatment. The primary end point of the study was disease-free survival. A second interim analysis revealed that a prespecified boundary for lack of benefit had been crossed.

\section{RESULTS}

At a median follow-up of 59 months, there was no significant between-group difference in the primary end point, with a rate of disease-free survival of $77 \%$ in each group (adjusted hazard ratio in the zoledronic acid group, 0.98; 95\% confidence interval [CI], 0.85 to $1.13 ; \mathrm{P}=0.79$ ). Disease recurrence or death occurred in 377 patients in the zoledronic acid group and 375 of those in the control group. The numbers of deaths - 243 in the zoledronic acid group and 276 in the control group - were also similar, resulting in rates of overall survival of $85.4 \%$ in the zoledronic acid group and $83.1 \%$ in the control group (adjusted hazard ratio, 0.85 ; 95\% CI, 0.72 to 1.01; $\mathrm{P}=0.07$ ). In the zoledronic acid group, there were 17 confirmed cases of osteonecrosis of the jaw (cumulative incidence, $1.1 \%$; $95 \% \mathrm{CI}, 0.6$ to $1.7 ; \mathrm{P}<0.001$ ) and 9 suspected cases; there were no cases in the control group. Rates of other adverse effects were similar in the two study groups.

\section{CONCLUSIONS}

These findings do not support the routine use of zoledronic acid in the adjuvant management of breast cancer. (Funded by Novartis Pharmaceuticals and the National Cancer Research Network; AZURE Current Controlled Trials number, ISRCTN79831382.) 
ETASTASIS IS A COMPLEX PROCESS THAT is dependent on both the biologic features of the primary tumor and cellular interactions within host tissues. In the bone microenvironment, cancer cells stimulate osteoblasts to release receptor activator of nuclear factor $\kappa \mathrm{B}$ ligand (RANKL), which binds to its receptor, RANK, on both precursor and mature osteoclasts. The resulting increase in osteoclastic bone resorption leads to the release of bone-derived growth factors that may provide a fertile environment for survival and growth of adjacent cancer cells. ${ }^{1}$ Thus, targeting bone-cell function provides a potential additional approach to preventing bone metastases as a component of standard adjuvant therapy. ${ }^{2}$ In many in vivo models, bisphosphonates prevent or delay metastasis. ${ }^{3}$ In addition, synergistic interactions between aminobisphosphonates and cytotoxic drugs have been shown in preclinical models. ${ }^{4,5}$

In patients with early-stage breast cancer, several clinical trials have suggested that the adjuvant use of bisphosphonates reduces rates of recurrence and death. ${ }^{6-8}$ In addition, despite a lack of regulatory approval in most health care systems, the inclusion of a bisphosphonate as part of adjuvant therapy has become increasingly widespread. In this randomized, controlled, open-label phase 3 study, called the Adjuvant Zoledronic Acid to Reduce Recurrence (AZURE) trial, we evaluated the adjuvant use of zoledronic acid in a broad population of patients with stage II or III early-stage breast cancer.

\section{METHODS}

\section{STUDY PATIENTS}

The research protocol and statistical analysis plan are available with the full text of this article at NEJM.org. To be eligible for the study, all the patients had to be at least 18 years of age, have a Karnofsky performance status of at least 80, and have a histologically confirmed breast cancer with axillary lymph-node metastasis (N1) or a T3-T4 primary tumor. Complete primary tumor resection was mandated or intended after neoadjuvant therapy. In addition, patients who were eligible for completion surgery (margin excision, mastectomy, or axillary lymph-node dissection) after completion of adjuvant chemotherapy could be included.

Patients were not eligible if there was clinical or imaging evidence of distant metastases or if complete treatment of the primary breast tumor and regional lymph nodes was not possible. Other exclusion criteria included a cancer diagnosis within the preceding 5 years, use of bisphosphonates during the previous year, or a diagnosis of osteoporosis or other bone disease likely to require bonetargeted treatment. The serum creatinine level had to be less than 1.5 times the upper limit of the normal range. In 2005, after case reports of osteonecrosis of the jaw associated with bisphosphonates, ${ }^{9}$ an amendment was adopted to exclude patients with clinically significant, active dental problems or planned jaw surgery.

\section{RANDOMIZATION AND TREATMENT}

After providing written informed consent, patients were randomly assigned in a 1:1 ratio to receive standard adjuvant systemic therapy (control group) or standard adjuvant systemic therapy along with zoledronic acid. The zoledronic acid was administered immediately after each cycle of adjuvant chemotherapy in a 4-mg dose by intravenous infusion every 3 to 4 weeks for 6 cycles and then every 3 months for 8 doses, followed by 5 cycles on a 6-month schedule for a total of 5 years. Dose adjustments for renal-function abnormalities were recommended in accordance with the product license. Daily oral supplements containing calcium (400 to $1000 \mathrm{mg}$ ) and vitamin D (200 to $500 \mathrm{IU}$ ) were recommended for all patients during the first 6 months and were continued thereafter at the discretion of the treating physician.

External-beam radiotherapy to the breast and chest wall, with or without irradiation of regional lymph nodes, and adjuvant cytotoxic and endocrine treatments were given in accordance with standard protocols at each participating institution. After regulatory approval of trastuzumab for adjuvant use, the drug was allowed in patients with HER2positive tumors.

To minimize imbalances in tumor and treatment characteristics between the study groups, the central, automated, 24-hour, computer-generated telephone randomization system incorporated the following variables: the number of involved axillary lymph nodes, clinical tumor stage, estrogen-receptor status, type and timing of systemic therapy, menopausal status, statin use or nonuse, and study center.

Prerandomization investigations in accordance with institutional protocols included histologic confirmation of breast cancer; testing of hematologic, renal, and hepatic function; and imaging 
for the purpose of tumor staging. The follow-up schedule for both the zoledronic acid group and the control group included clinical assessment, physical examination, monitoring for adverse events, and measurement of hematologic, renal, and hepatic function. Investigations for possible recurrence were clinically directed as deemed appropriate by the treating physician. Routine followup imaging was not mandated.

The study treatment was discontinued in the event of a distant recurrence, unacceptable toxicity, three consecutively missed treatments, the patient's request, the treating physician's recommendation, or completion of 5 years of treatment. Continuation of the study medication was recommended in the event of a locoregional recurrence and at the physician's discretion after the diagnosis of a new primary cancer.

\section{STUDY END POINTS}

The primary end point of the study was disease-free survival, which was defined as an absence of distant recurrence, of any invasive locoregional recurrence (except for ipsilateral operable relapse within a conserved breast), and of death from any cause without recurrence. The secondary end point was overall survival. Prespecified subgroup analyses were based on variables included in the randomization.

We also performed exploratory analyses to investigate treatment effects on sites of first recurrence. After a protocol amendment was approved before the first interim analysis in 2008, we added another secondary end point: invasive-disease-free survival, which was defined according to the Standardized Definitions for Efficacy and End Points in Adjuvant Breast Cancer Trials (STEEP) guidelines. ${ }^{10}$ (A complete definition is provided in the Supplementary Appendix, available at NEJM.org.) The date of recurrence was defined as the date on which relapse was first suspected, rather than the date on which it was confirmed, to reduce the risk of ascertainment bias. On-site and telephone-based monitoring was performed to ensure that recurrence dates were backdated to the date on which the event was first suspected, when this date preceded clinical, histologic, or imaging confirmation. ${ }^{11}$

\section{STUDY OVERSIGHT}

The study was sponsored by the University of Sheffield and approved as a United Kingdom national trial by the Clinical Trials Advisory Awards
Committee. Grant support was provided by Novartis Pharmaceuticals and was supplemented in the United Kingdom by the infrastructure of the National Cancer Research Network. Novartis Pharmaceuticals donated study supplies of zoledronic acid. The authors developed the study concept, wrote the protocol, and performed and reviewed all analyses. The study was conducted in accordance with the protocol, with amendments to reduce the risk of osteonecrosis of the jaw and to inform both patients and dental practitioners of this risk. The first author wrote the first draft of the manuscript, and all authors were involved in revision and approval of the manuscript. Novartis Pharmaceuticals was given an opportunity to comment on the manuscript, but all decisions on submission of the manuscript for publication were made by the authors and the trial steering committee.

\section{STATISTICAL ANALYSIS}

A final analysis was planned after the primary end point (disease-free survival) had occurred in 940 patients, on the basis of the recruitment of 3300 patients during a 3-year period, an anticipated rate of disease-free survival of $75 \%$ at 3 years, and a $5 \%$ annual rate of loss to follow-up. It was estimated that these numbers would provide a power of $80 \%$ to detect a relative reduction of $17 \%$ in the rate of disease recurrence or death among patients receiving zoledronic acid, at a two-sided level of significance of 0.05 , which would approximate an absolute benefit of 3.7 percentage points.

A single interim analysis was planned after the primary end point had been reached in 470 patients, with a two-sided alpha level of $0.005 .{ }^{12}$ After this analysis was performed, on the advice of the independent data and safety monitoring committee, no efficacy data were released. Because the rate of events contributing to the primary end point was lower than predicted (resulting in a combined rate of disease-free survival of $85 \%$ at 3 years), an independent statistician who was unaware of the findings and was not involved in the first interim analysis provided revised stopping boundaries for both efficacy and lack of benefit that would allow timely release of a clinically important result. A second interim analysis was planned after the primary end point had occurred in at least 705 patients, along with a $0.5 \%$ probability of declaring false positive results (one-sided) or a $5.0 \%$ probability of declaring negative results with the use of a group 


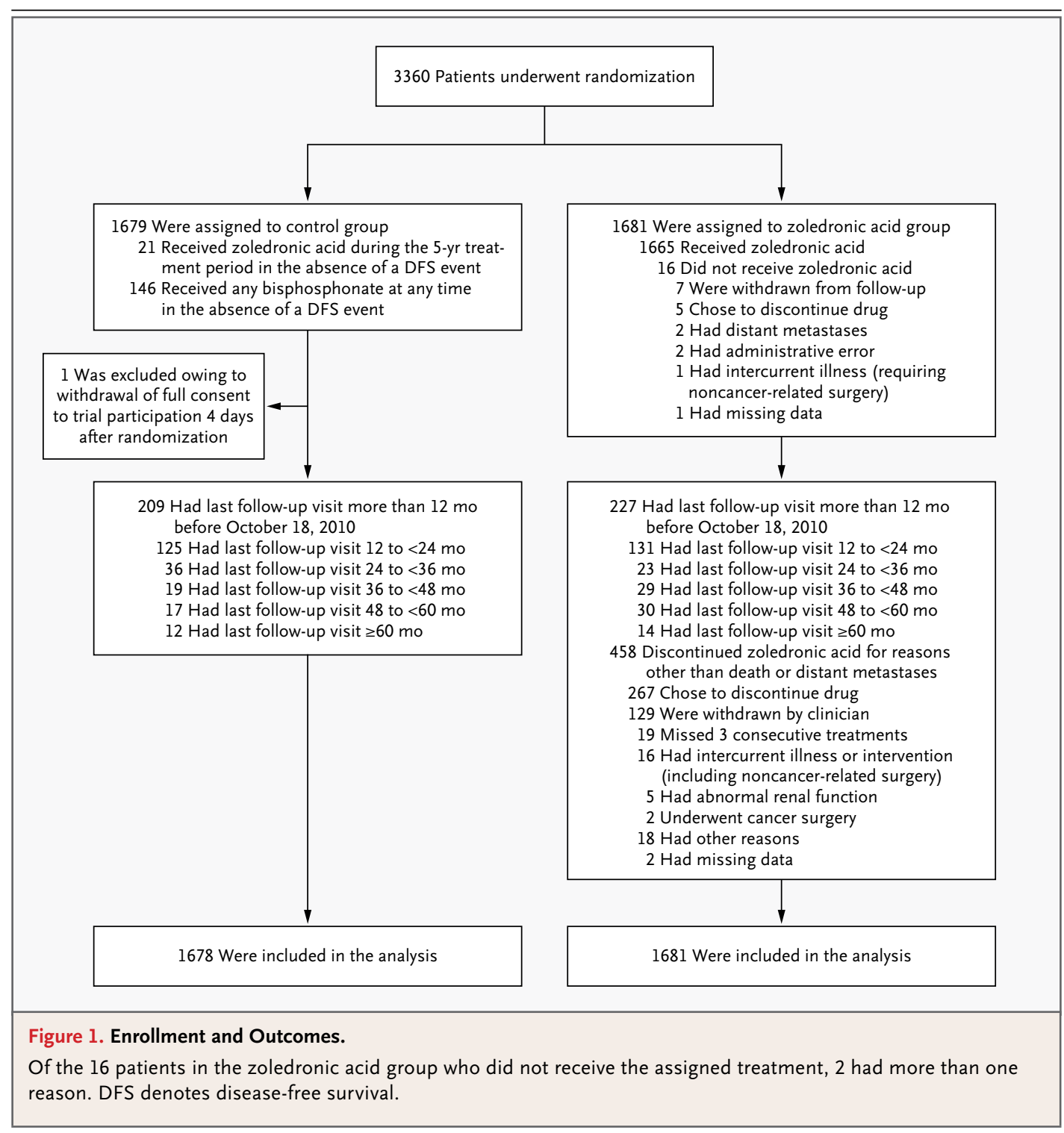

sequential-design method. ${ }^{13}$ The analysis was carried out on 752 events, resulting in an efficacy boundary for the hazard ratio of 0.833 (lower boundary) and a lack-of-efficacy boundary of 0.936 (upper boundary). At this interim analysis, the lack-of-efficacy boundary was crossed, and the committee recommended the release of results.

We used Kaplan-Meier survival curves to evaluate the rates of disease-free survival, invasivedisease-free survival, and overall survival. We compared between-group differences using the log-rank test and a Cox proportional-hazards model to adjust for the minimization factors (excluding study center). We calculated rates of osteonecrosis of the jaw using cumulative incidence functions, in which deaths without a diagnosis of osteonecrosis of the jaw were considered to be competing-risk events and were compared with the use of the log-rank test. In a subgroup analysis, we used the Cox proportional-hazards model to adjust for statistically significant factors in the overall analysis (estrogen-receptor status, lymphnode involvement, and tumor stage).

All adverse events were evaluated in the safety population. No statistical testing was carried out on these end points, apart from confirmed cases of osteonecrosis of the jaw, since this disorder had been identified a priori as a clinically important event linked to bisphosphonate use. All analyses were performed with the use of SAS software, version 9.2 (SAS Institute). 


\begin{tabular}{|c|c|c|}
\hline Characteristic & $\begin{array}{l}\text { Zoledronic Acid } \\
\qquad(N=1681)\end{array}$ & $\begin{array}{l}\text { Control } \\
(\mathrm{N}=1678)\end{array}$ \\
\hline \multicolumn{3}{|l|}{ Axillary lymph nodes — no. (\%) } \\
\hline 0 & $29(1.7)$ & $32(1.9)$ \\
\hline $1-3$ & $1041(61.9)$ & $1032(61.5)$ \\
\hline$\geq 4$ & $604(35.9)$ & $608(36.2)$ \\
\hline Unknown & $7(0.4)$ & $6(0.4)$ \\
\hline \multicolumn{3}{|l|}{ Tumor stage - no. (\%) } \\
\hline $\mathrm{Tl}$ & $542(32.2)$ & $523(31.2)$ \\
\hline $\mathrm{T} 2$ & $851(50.6)$ & $867(51.7)$ \\
\hline $\mathrm{T} 3$ & $227(13.5)$ & $228(13.6)$ \\
\hline $\mathrm{T} 4$ & $58(3.5)$ & $59(3.5)$ \\
\hline $\mathrm{TX}$ & $3(0.2)$ & $1(0.1)$ \\
\hline \multicolumn{3}{|l|}{ Estrogen-receptor status - no. (\%) } \\
\hline Positive & $1319(78.5)$ & $1316(78.4)$ \\
\hline Negative & $349(20.8)$ & $355(21.2)$ \\
\hline Unknown & $13(0.8)$ & $7(0.4)$ \\
\hline \multicolumn{3}{|l|}{ Menopausal status - no. (\%) } \\
\hline Premenopausal & $751(44.7)$ & $752(44.8)$ \\
\hline \multicolumn{3}{|l|}{ Postmenopausal } \\
\hline$\leq 5 \mathrm{yr}$ & $247(14.7)$ & $244(14.5)$ \\
\hline$>5 \mathrm{yr}$ & $519(30.9)$ & $522(31.1)$ \\
\hline Status unknown & $164(9.8)$ & $160(9.5)$ \\
\hline \multicolumn{3}{|l|}{ Planned systemic therapy — no. (\%) } \\
\hline Endocrine therapy alone & $76(4.5)$ & $75(4.5)$ \\
\hline Chemotherapy alone & $362(21.5)$ & $360(21.5)$ \\
\hline Endocrine therapy plus chemotherapy & $1243(73.9)$ & $1243(74.1)$ \\
\hline \multicolumn{3}{|l|}{$\begin{array}{c}\text { Planned type of chemotherapy - no./ } \\
\text { total no. (\%) }\end{array}$} \\
\hline Anthracyclines & $1567 / 1605$ (97.6) & $1564 / 1603(97.6)$ \\
\hline Taxanes & $390 / 1605(24.3)$ & $385 / 1603(24.0)$ \\
\hline \multicolumn{3}{|l|}{$\begin{array}{c}\text { Timing of chemotherapy - no./ } \\
\text { total no. (\%) }\end{array}$} \\
\hline Neoadjuvant & $104 / 1605(6.5)$ & $104 / 1603(6.5)$ \\
\hline Postoperative & $1501 / 1605$ (93.5) & $1499 / 1603(93.5)$ \\
\hline Statin use - no. (\%) & $97(5.8)$ & $101(6.0)$ \\
\hline
\end{tabular}

* There were no significant differences between the two groups. assigned to receive zoledronic acid, 1665 (99.0\%) received at least one dose (Fig. 1). The tumor and treatment characteristics of the patients in the two study groups were well balanced (Table 1). A total of $95.5 \%$ of the patients received chemotherapy, which was planned to include an anthracycline in $97.6 \%$ of the patients and a taxane in $24.2 \%$; 208 patients received neoadjuvant chemotherapy and 3000 received postoperative chemotherapy.

When the database was locked, on October 18, 2010, the median follow-up period was 59.3 months (interquartile range, 53.5 to 60.9 ) in the zoledronic acid group and 58.6 months (interquartile range, 52.7 to 60.9 ) in the control group. The median time since the last follow-up contact for all surviving patients was 5.9 months (interquartile range, 3.8 to 9.1), which was identical in the two groups.

\section{PRIMARY AND SECONDARY END POINTS}

At 5 years, $76.9 \%$ and $77.1 \%$ of the patients in the treatment and control groups, respectively, were alive and disease-free; 377 patients in the zoledronic acid group and 375 patients in the control group had recurrent disease or had died (adjusted hazard ratio in the zoledronic acid group, $0.98 ; 95 \%$ confidence interval [CI], 0.85 to $1.13 ; \mathrm{P}=0.79$ ) (Fig. 2A). Similarly, at 5 years, $75.4 \%$ and $75.3 \%$ of the patients in the treatment and control groups, respectively, were alive and free of invasive disease; 404 patients in the zoledronic acid group and 403 patients in the control group had invasive disease or had died (adjusted hazard ratio, 0.98; 95\% CI, 0.85 to 1.12; $\mathrm{P}=0.73$ ) (Fig. 2B). The distributions of invasive-disease events were also similar in the two groups; $65.8 \%$ were distant recurrences, of which 230 were in bone (108 in the zoledronic acid group and 122 in the control group) (Table 1 in the Supplementary Appendix).

Despite the known effects of many cancer treatments on bone health and the risk of osteoporosis, only 146 patients in the control group $(8.7 \%)$ received a bisphosphonate before reaching the primary end point. To date, 519 patients have died: 243 in the zoledronic acid group (5-year overall survival rate, $85.4 \%$ ) and 276 in the control group (5-year overall survival rate, 83.1\%) (adjusted hazard ratio with zoledronic acid, 0.85 ; $95 \% \mathrm{CI}$, 0.72 to $1.01 ; \mathrm{P}=0.07$ ).

\section{STUDY PATIENTS}

RESULTS

Between September 2003 and February 2006, a total of 3360 eligible women from 174 centers in seven countries were recruited. Of these patients, 1681 were assigned to the zoledronic acid group and 1679 to the control group. Among the patients

\section{SUBGROUP ANALYSES}

Prespecified subgroup analyses showed a consistent lack of differential effect across the variables that were included in the randomization, with the 
exception of menopausal status. Significant heterogeneity of treatment effect $\left(\chi^{2}{ }_{1}=7.91, \mathrm{P}=0.005\right)$ on the rate of invasive-disease-free survival was observed between patients who had undergone menopause more than 5 years earlier and all other patients (those who were premenopausal or perimenopausal and those with unknown menopausal status). At 5 years, among postmenopausal patients, the rates of invasive-disease-free survival were $78.2 \%$ in the zoledronic acid group and $71.0 \%$ in the control group (adjusted hazard ratio with zoledronic acid, $0.75 ; 95 \% \mathrm{CI}, 0.59$ to $0.96 ; \mathrm{P}=0.02$ ); among all other patients, the rates were $74.1 \%$ in the zoledronic acid group and $77.2 \%$ in the control group (adjusted hazard ratio, 1.15; 95\% CI, 0.97 to 1.36; $\mathrm{P}=0.11$ ) (Fig. $3 \mathrm{~A}$ and $3 \mathrm{~B}$ ). In addition, among patients who had undergone menopause more than 5 years earlier, the 5 -year overall survival rate was $84.6 \%$ in the zoledronic acid group and $78.7 \%$ in the control group (adjusted hazard ratio, 0.74; $95 \%$ CI, 0.55 to $0.98 ; \mathrm{P}=0.04$ ), as compared with all other patients, for whom the rates were $85.7 \%$ in the zoledronic acid group and $85.1 \%$ in the control group (adjusted hazard ratio, 0.97; 95\% CI, 0.78 to $1.21 ; \mathrm{P}=0.81$ ) (Fig. $3 \mathrm{C}$ and $3 \mathrm{D}$ ). These differences were independent of estrogen-receptor status, tumor stage, and lymph-node involvement.

With respect to distant skeletal recurrence, the effects of zoledronic acid did not differ significantly between the two menopausal groups $\left(\chi^{2}{ }_{1}=0.14, \mathrm{P}=0.70\right)$. In contrast, for the other components of invasive-disease-free survival, there was a consistent, significant difference in the effects of zoledronic acid according to menopausal status, with an apparent benefit in postmenopausal women and potential harm in all other women $\left(\chi^{2}{ }_{1}=14.00, P<0.001\right)$ (Fig. 1 in the Supplementary Appendix).

\section{ADVERSE EVENTS}

Dental adverse events were more frequent in the zoledronic acid group than in the control group, and investigators reported 26 cases of osteonecrosis of the jaw. Of these cases, 17 were confirmed on central review, all in the zoledronic acid group (rate, $1.1 \%$; $95 \% \mathrm{CI}, 0.6$ to 1.7 ), including 3 cases occurring after metastases to bone. Of the 9 unconfirmed cases, 4 did not conform to a standard definition of osteonecrosis of the jaw, and further information is awaited on 5 cases. The rate of fracture was reduced in patients receiving zoledronic acid, with 175 fractures (65 in the zoledronic acid group and 110 in the control group) in 152 patients

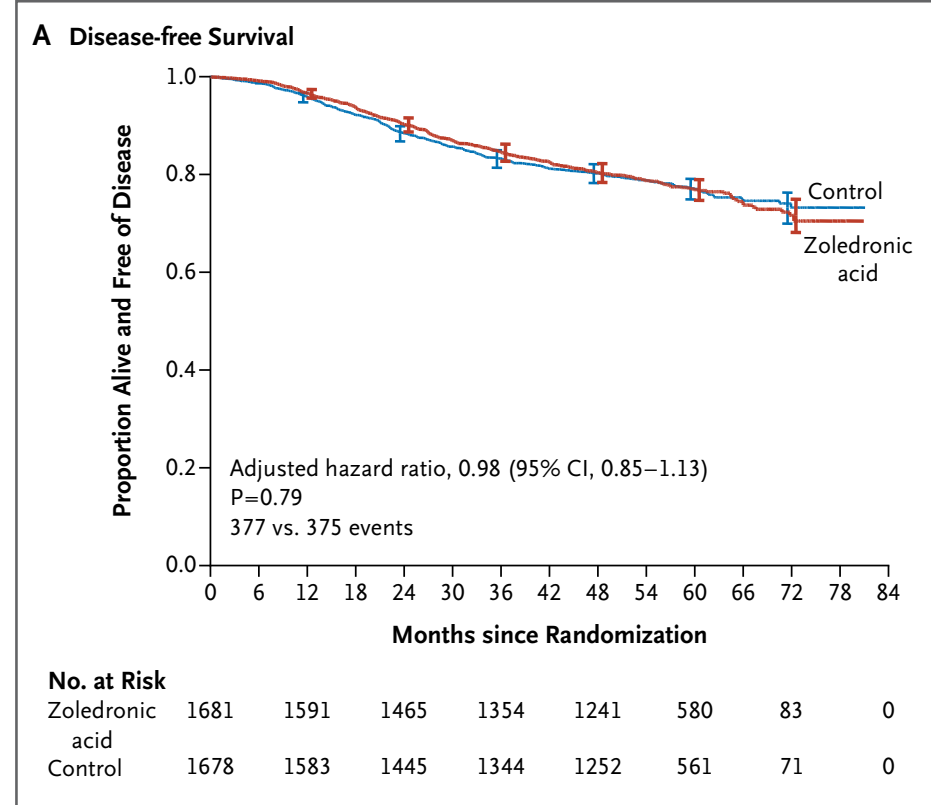

B Invasive-Disease-free Survival

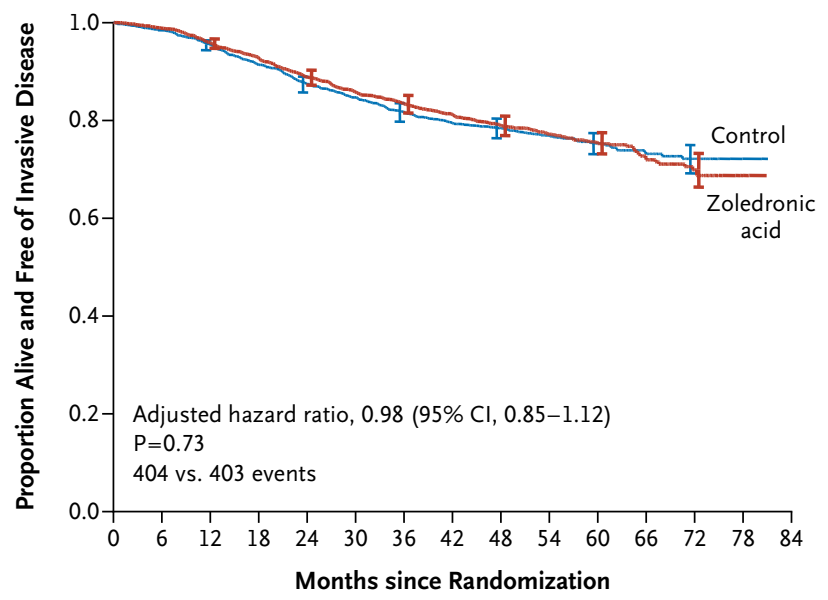

No. at Risk

$\begin{array}{ccccccccc}\begin{array}{c}\text { Zoledronic } \\ \text { acid }\end{array} & 1681 & 1578 & 1443 & 1337 & 1222 & 570 & 82 & 0 \\ \begin{array}{c}\text { Control } \\ 1678\end{array} & 1574 & 1426 & 1316 & 1221 & 544 & 68 & 0\end{array}$

Figure 2. Disease-free Survival and Invasive-Disease-free Survival in the Intention-to-Treat Population.

Panel A shows the proportion of patients who were alive without a recurrence of disease, and Panel $B$ shows the proportion of patients who were alive without invasive disease at 5 years and beyond. The I bars represent $95 \%$ confidence intervals.

(60 in the zoledronic acid group and 92 in the control group). Rates of other adverse events were similar in the two study groups (Table 2, and Tables 2 and 3 in the Supplementary Appendix). Most serious adverse events were related to chemotherapy or other cancer treatments. No significant difference was seen in rates of neutro- 


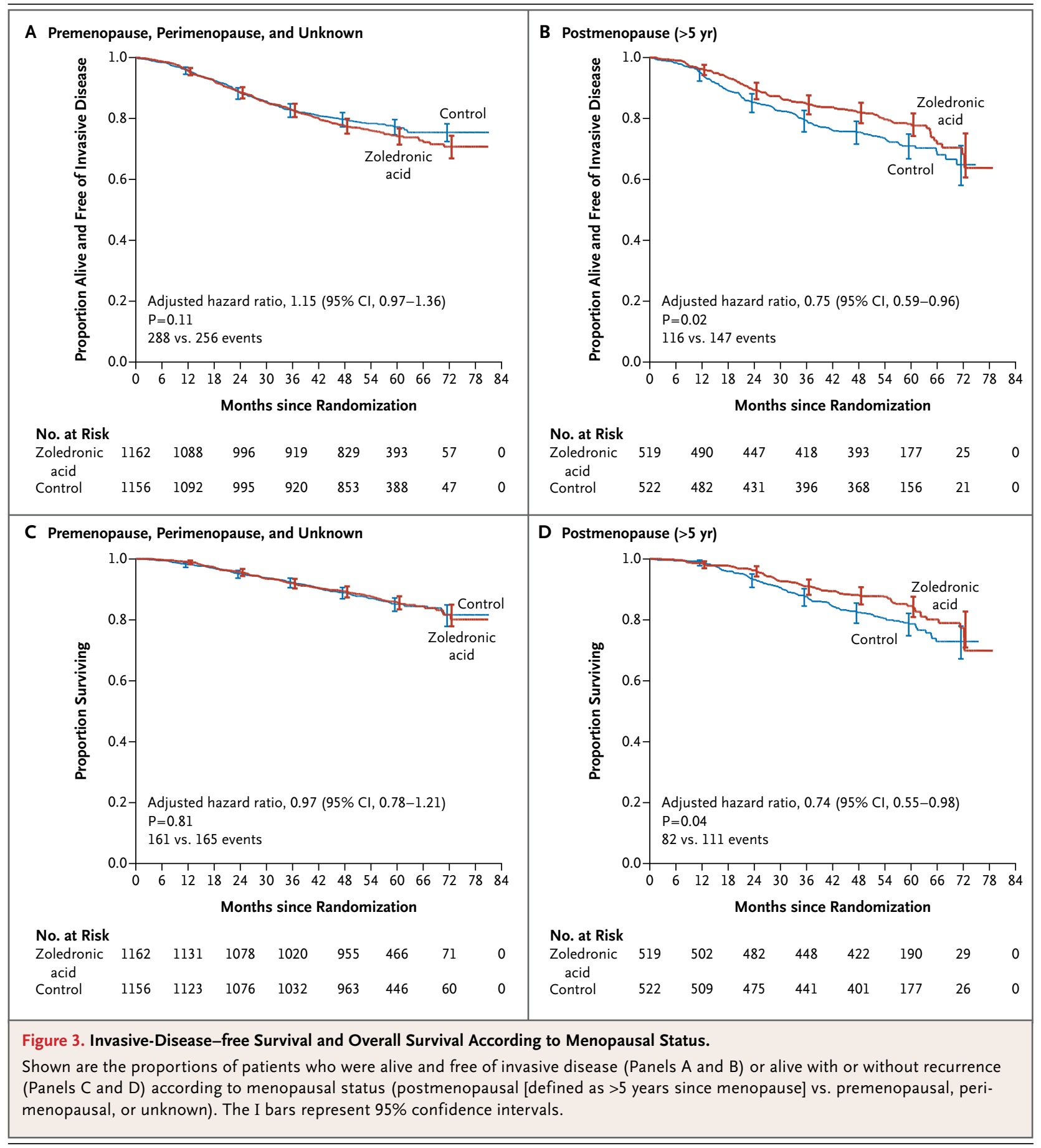

penic fever. Chemotherapy dose reductions and delays were similar in the two study groups (data not shown).

\section{DISCUSSION}

Clinical trials of adjuvant bisphosphonates in patients with early-stage breast cancer have had variable results. A reduction in bone metastases and improved overall survival were reported in two trials of oral clodronate. ${ }^{6,7}$ However, two other trials showed no benefit. ${ }^{14,15}$ More recently, in the Austrian Breast and Colorectal Cancer Study Group 12 (ABCSG-12) trial (ClinicalTrials.gov number, NCT00295646) involving 1803 premenopausal women with estrogen-receptor-positive breast 


\begin{tabular}{|c|c|c|c|}
\hline \multirow[t]{2}{*}{ Serious Adverse Event } & $\begin{array}{l}\text { Zoledronic Acid } \\
\qquad(\mathrm{N}=1686)\end{array}$ & $\begin{array}{l}\text { Control } \\
(\mathrm{N}=1666)\end{array}$ & $\begin{array}{l}\text { Difference } \\
(95 \% \mathrm{Cl}) \dagger\end{array}$ \\
\hline & \multicolumn{2}{|c|}{ number of patients (percent) } & percentage points \\
\hline \multicolumn{4}{|l|}{ Neutropenia } \\
\hline Septic & $160(9.5)$ & $159(9.5)$ & 0.1 ( -2.0 to 1.9$)$ \\
\hline Any & $41(2.4)$ & 49 (2.9) & $-0.5(-1.6$ to 0.6$)$ \\
\hline Pyrexia & $37(2.2)$ & $24(1.4)$ & $0.8(-0.1$ to 1.7$)$ \\
\hline Vomiting & $35(2.1)$ & $23(1.4)$ & $0.7(-0.2$ to 1.6$)$ \\
\hline Lower respiratory infection & $24(1.4)$ & $33(2.0)$ & $-0.6(-1.4$ to 0.3$)$ \\
\hline Central-catheter infection & $24(1.4)$ & $21(1.3)$ & $0.2(-0.6$ to 0.9$)$ \\
\hline Cellulitis & $21(1.2)$ & $21(1.3)$ & $0.0(-0.8$ to 0.7$)$ \\
\hline Pulmonary embolus: & $25(1.5)$ & $13(0.8)$ & 0.7 (0.0 to 1.4$)$ \\
\hline \multicolumn{4}{|l|}{ Osteonecrosis of the jaw } \\
\hline Confirmed』 & $17(1.1)$ & 0 & NA \\
\hline Suspected & 9 & 0 & NA \\
\hline \multicolumn{4}{|c|}{$\begin{array}{l}\text { * Listed are serious adverse events that occurred in more than } 1 \% \text { of patients in either study group in the safety popula- } \\
\text { tion, which is defined in the Supplementary Appendix. Adverse events that have been classified according to organ sys- } \\
\text { tem and grade are listed in Tables } 2 \text { and } 3 \text { in the Supplementary Appendix. NA denotes not applicable. } \\
\dagger \text { Positive values indicate a greater frequency of the event in the zoledronic-acid group. Between-group differences were } \\
\text { calculated before the rounding of percentages. } \\
t \text { In addition to the cases of pulmonary embolus that were classified as serious adverse events, } 3 \text { cases ( } 1 \text { in the zole- } \\
\text { dronic-acid group and } 2 \text { in the placebo group) were reported as adverse events, for a total of } 41 \text { patients with pulmo- } \\
\text { nary embolus ( } 1.5 \% \text { in the zoledronic acid group and } 0.9 \% \text { in the control group; absolute difference, } 0.6 \text { percentage } \\
\text { points; } 95 \% \text { confidence interval, }-0.1 \text { to } 1.4) \text {. } \\
\int P<0.001 .\end{array}$} \\
\hline
\end{tabular}

cancer who were treated with goserelin and either tamoxifen or anastrozole, the administration of zoledronic acid every 6 months for 3 years reduced the risk of disease recurrence by about one third. ${ }^{8}$ In addition, several large randomized trials addressing the question have completed accrual and are in the follow-up phase.

In our study, no improvement was seen in the rate of disease-free survival, the primary end point of the study; rates of invasive-disease-free survival and overall survival were similar in the two study groups. This primary result is unlikely to change with further follow-up and appears to differ markedly from the findings of Gnant and colleagues in the ABCSG-12 study. ${ }^{8}$ However, there are important differences between the two study populations. In the ABCSG-12 study, all the patients started receiving goserelin and endocrine therapy (resulting in a rapid suppression of reproductive hormones) before the initiation of bisphosphonate treatment; in addition, the patients had disease with a good prognosis, and less than $5 \%$ received chemotherapy. In contrast, the premenopausal patients in our study had a less favorable prognosis, more than 95\% received chemotherapy, and all had premenopausal levels of reproductive hormones at study entry. Only three of the premenopausal patients $(0.2 \%)$ in our study received goserelin.

In a prespecified analysis, our finding of a possible benefit of zoledronic acid in patients who had undergone menopause more than 5 years before study entry is intriguing. From an endocrine perspective, the postmenopausal patients in our study were similar to the goserelin-treated patients in the ABCSG-12 study, ${ }^{8}$ who had low levels of reproductive hormones at study entry. The curves for rates of disease-free survival in the postmenopausal subgroup diverged rapidly (absolute difference, 3 percentage points at 1 year and 5 percentage points at 2 years) and showed a small but significant survival advantage for patients who received zoledronic acid. Furthermore, the use of zoledronic acid appeared to have divergent effects on metastasis to visceral and locoregional sites according to menopausal status. Our analysis of the interaction of the various components of invasive-disease-free survival suggests a systemic effect of zoledronic 
acid that operates differently according to menopausal status and that is distinct from any effect in bone (Fig. 1 in the Supplementary Appendix). The explanation for this finding is unclear, but perhaps bone provides a sanctuary for cancer cells, and after treatment with zoledronic acid, the ability of cancer cells to disseminate to other body sites is dependent on the presence of reproductive hormones.

The apparent early benefits seen in the postmenopausal subgroup suggest that the initial phase of treatment, when zoledronic acid is combined with chemotherapy, may be most important. During neoadjuvant chemotherapy with zoledronic acid, an increased tumor response in the breast $^{16}$ and a reduced number of disseminated tumor cells in the bone marrow ${ }^{17}$ have been observed, suggesting positive interactions between zoledronic acid and chemotherapy.

Adjuvant chemotherapy would be expected to induce menopause in a high proportion of women over the age of 40 years. However, it may take many months for chemotherapy to affect reproductive hormone levels, and despite causing amenorrhea, chemotherapy may not result in complete ovarian suppression. It is unclear how the endocrine environment in bone might influence interactions between chemotherapy and zoledronic acid. However, as levels of ovarian-derived inhibin decline after menopause, the control of bone-cell function changes from primary regulation through effects of inhibins on osteoblasts to control by locally produced activin interacting with bone morphogenic proteins and noggin to drive bone turnover. ${ }^{18}$

In our study, zoledronic acid had no demonstrable effect on chemotherapy-associated toxic effects. A full description of the adverse-event profile in this trial has been reported previously. ${ }^{19}$ Confirmed osteonecrosis of the jaw occurred in $1.1 \%$ of patients who were treated with zole- dronic acid, with an additional nine suspected cases. Resolution of osteonecrosis of the jaw was seen in some cases, but the observed frequency of this complication in our study was higher than that reported in other studies of zoledronic acid in patients receiving adjuvant therapy.8,20 This discrepancy presumably reflects the more intensive schedule of zoledronic acid and greater use of chemotherapy in our study. On the positive side, zoledronic acid was associated with a reduction in rates of fracture, particularly among patients who had a disease recurrence.

In conclusion, our findings do not support the routine use of zoledronic acid as adjuvant therapy in unselected patients with early-stage breast cancer. Further investigation into the possible interaction between zoledronic acid and reproductive hormones is required. For postmenopausal women, the use of bisphosphonates remains appropriate for the prevention of treatment-induced bone loss and osteoporosis and might have beneficial effects on disease outcomes. The optimum schedule, duration, and type of bisphosphonate therapy remain unknown.

Supported by a grant from Novartis Pharmaceuticals and by the National Cancer Research Network.

Disclosure forms provided by the authors are available with the full text of this article at NEJM.org.

We thank the patients who took part in this trial; all investigators and research staff at the 174 recruiting centers; the staff at the clinical trials research units in the United Kingdom (Clinical Trials Research Unit [CTRU], University of Leeds; Institute of Cancer Research [ICR], Sutton; Edinburgh Clinical Trials Unit; and Cancer Research U.K. Clinical Trials Unit, University of Birmingham), Australia (Victorian Cooperative Oncology Group), Ireland (Irish Clinical Oncology Research Group), and Spain (Spanish Group of Treatment Study and Other Experiences in Solid Tumors); Michelle Collinson (CTRU) and Roger A'Hern (ICR) for their statistical input; the members of the trial steering committee under the chairmanship of Prof. Matthew Seymour; and the members of the independent data and safety monitoring committee (Dr. Laurence Collette, Prof. Malcolm Mason, and Dr. Richard Theriault).

The authors' affiliations are as follows: the Academic Unit of Clinical Oncology, Weston Park Hospital, University of Sheffield (for the National Cancer Research Network and National Institute for Health Research) (R.E.C., R. Burkinshaw); Clinical Trials Research Unit (H.M., C.D., V.H., W.G.) and St. James Institute of Oncology (D.D.), University of Leeds, Leeds; Western General Hospital, University of Edinburgh, Edinburgh (D.C.); St. Luke's Cancer Centre, Royal Surrey County Hospital, Guildford (S.J.H.); University Hospitals Coventry and Warwickshire National Hospital Service Trust, Coventry (R.J.G.); Velindre Cancer Center, Whitchurch, Cardiff (P.J.B.-L.); Beatson West of Scotland Cancer Centre, Glasgow (D.R.); Institute of Cancer Research Clinical Trials Support Unit, Sutton (J.P.); Cancer Research United Kingdom Clinical Trials Unit, University of Birmingham, Birmingham (C.G.); and Cancer Clinical Trials Team, National Health Service National Services Scotland, Edinburgh (U.R.) - all in the United Kingdom; University Hospital Galway, Galway, Ireland (for the All Ireland Cooperative Oncology Research Group) (M.K.); Institut Català d'Oncologia-Institut d'Investigació Biomèdica de Bellvitge, L'Hospitalet de Llobregat, Barcelona (for the Spanish Group of Treatment Study and Other Experiences in Solid Tumors) (M.G.); and Victoria Cooperative Oncology Group, Cancer Council Victoria, Carlton (J.P.); and Andrew Love Cancer Centre, Geelong (for the Victoria Cooperative Oncology Group) (R. Bell) — both in Australia. 
REFERENCES

1. Mundy GR. Metastasis to bone: causes, consequences and therapeutic opportunities. Nat Rev Cancer 2002;2:584-93.

2. Woodward EJ, Coleman RE. Prevention and treatment of bone metastases. Curr Pharm Des 2010;16:2998-3006.

3. Neville-Webbe HL, Coleman RE. Bisphosphonates and RANK ligand inhibitors for the treatment and prevention of metastatic bone disease. Eur J Cancer 2010; 46:1211-22.

4. Ottewell PD, Mönkkönen H, Jones M, et al. Antitumor effects of doxorubicin followed by zoledronic acid in a mouse model of breast cancer. J Natl Cancer Inst 2008;100:1167-78.

5. Ottewell PD, Deux B, Monkkonen H et al. Differential effect of doxorubicin and zoledronic acid on intraosseous versus extraosseous breast tumor growth in vivo. Clin Cancer Res 2008;14:4658-66.

6. Diel IJ, Jaschke A, Solomayer EF, et al. Adjuvant oral clodronate improves the overall survival of primary breast cancer patients with micrometastases to the bone marrow: a long-term follow-up. Ann Oncol 2008;19:2007-11.

7. Powles T, Paterson A, McCloskey E, et al. Reduction in bone relapse and improved survival with oral clodronate for adjuvant treatment of operable breast cancer. Breast Cancer Res 2006;8:R13.

8. Gnant M, Mlineritsch B, Schippinger W, et al. Endocrine therapy plus zoledronic acid in premenopausal breast cancer. $\mathrm{N}$ Engl J Med 2009;360:679-91.

9. Marx RE. Pamidronate (Aredia) and zoledronate (Zometa) induced avascular necrosis of the jaws: a growing epidemic. J Oral Maxillofac Surg 2003;61:1115-7.

10. Hudis CA, Barlow WE, Costantino JP, et al. Proposal for standardized definitions for efficacy end points in adjuvant breast cancer trials: the STEEP system. J Clin Oncol 2007;25:2127-32.

11. European Organisation for Research and Treatment of Cancer. Manual for clinical research in breast cancer. 5th ed. London: Greenwich Medical Media, 2004. 12. O'Brien PC, Fleming TR. A multiple testing procedure for clinical trials. Biometrics 1979;35:549-56.

13. Whitehead J. Group sequential trials revisited: simple implementation using SAS. Stat Methods Med Res 2010 September 27 (Epub ahead of print).

14. Saarto T, Vehmanen L, Virkkunen P, Blomqvist C. Ten-year follow-up of a randomized controlled trial of adjuvant clodronate treatment in node-positive breast cancer patients. Acta Oncol 2004; 43:650-6.

15. Kristensen B, Ejlertsen B, Mouridsen $\mathrm{HT}$, et al. Bisphosphonate treatment in primary breast cancer: results from a randomised comparison of oral pamidronate versus no pamidronate in patients with primary breast cancer. Acta Oncol 2008; 47:740-6.

16. Coleman RE, Winter MC, Cameron D, et al. The effects of adding zoledronic acid to neoadjuvant chemotherapy on tumour response: exploratory evidence for direct anti-tumour activity in breast cancer. Br J Cancer 2010;102:1099-105.

17. Aft $R$, Naughton $M$, Trinkaus $K$, et al. Effect of zoledronic acid on disseminated tumour cells in women with locally advanced breast cancer: an open label, randomised, phase 2 trial. Lancet Oncol 2010; 11:421-8.

18. Nicks KM, Fowler TW, Akel NS, et al. Bone turnover across the menopause transition: the role of gonadal inhibins. Ann N Y Acad Sci 2010;1192:153-60.

19. Coleman R, Woodward E, Brown J, et al. Safety of zoledronic acid and incidence of osteonecrosis of the jaw (ONJ) during adjuvant therapy in a randomised phase III trial (AZURE: BIG 01-04) for women with stage II/III breast cancer. Breast Cancer Res Treat 2011;127:429-38.

20. Eidtmann $H$, de Boer R, Bundred N, et al. Efficacy of zoledronic acid in postmenopausal women with early breast cancer receiving adjuvant letrozole: 36-month results of the ZO-FAST study. Ann Oncol 2010;21:2188-94.

Copyright (c) 2011 Massachusetts Medical Society.

To receive the table of contents of the Journal by e-mail every Wednesday evening, sign up at NEJM.org. 